\title{
Estrutura teórico-metodológica de participação habilitadora de usuários na Estratégia Saúde da Família
}

Maria de Fátima Antero Sousa Machado' Neiva Francenely Cunha Vieira ${ }^{2}$

Este estudo tem como objetivo apresentar um construto teórico denominado Estrutura Teórico-Metodológica de uma Participação Habilitadora, proposta para compreender a participação de usuários na Estratégia Saúde da Família. A estrutura nos auxiliou a compreender o tipo de participação do usuário dentro da Estratégia Saúde da Família no sentido de indicar, por intermédio dos estágios de mudança, qual a tendência teórico-metodológica presente nas ações educativas.

Descritores: Estratégia Saúde da Família, Participação, Educação em Saúde.

\section{Theoretic methodological structure of enabling participation of Family Health Strategy users}

This paper aims to present a theoretical construct named theoretical methodological structure of an enabling participation, proposed to comprehend the participation of users in Family Health Strategy. The structure helped us to understand the kind of user's participation in health family strategy, indicating by means of changings levels, what is the theoretical methodology tendency that is present in education activities.

Descriptors: Family Health Strategy, Participation, Health Education.

\section{Estructura teórico metodológica de la participación habilitadora de usuários en la Estratégia de Salud de la Familia}

Esta investigación tiene por objetivo presentar un constructo teorico llamado estructura teorico metodologico de una participación habiliatadora, propuesta para comprender la participación de los usuários en la estratégia de la salud de la familia. La estructura nos ayudó a comprender el tipo de participación del usuário dentro de la estratégia salud de la familia de modo a indicar, por intermédio de los estágios de alteraciones, cual es la tendência teórico metodológica que está presente en las acciones educativas.

Descriptores: Estrategia Salud de la Familia, Participación, Educación en Salud.

\section{INTRODUÇÃO}

A adoção de modelos e teorias para a investigação deve ocorrer no sentido de nortear a investigação nas análises e, consequentemente, a produção do conhecimento.

A utilização de modelos e teorias para a investigação no campo da promoção da saúde pode facilitar o entendimento dos determinantes dos problemas de saúde e orientar nas soluções que respondam às necessidades e aos interesses das pessoas envolvidas. $\mathrm{O}$ uso de modelos e teorias como suporte para orientar a prática contribui para que o planejamento de ações se aproxime do contexto da clientela, além de ajudar a compreender as dificuldades e limitações dos programas de promoção da saúde ${ }^{(1)}$.

Modelo é um grupo de conceitos sistematicamente constituídos, cientificamente embasados e logicamente relacionados, o qual identifica componentes essenciais da prática, denominado também de modelo conceitual ou estrutura conceitual. Teorias e modelos são bases para investigação ${ }^{(2,3)}$. É o caminho para identificar um fenômeno, para apontar aqueles componentes ou características que dão identidade ao fenômeno e dar suporte à prática profissional ${ }^{(4)}$.

A estrutura conceitual, portanto, é um paradigma ou elenco de conceitos e proposições gerais e abstratas que fornecem uma estrutura de referência em um domínio de investigação de uma disciplina que trabalha com os conceitos ${ }^{(5)}$.

Assim, modelo é entendido como referenciais conceituais, representações elaboradas de algum aspecto do ambiente, utilizando as abstrações como blocos embasadores; e teoria é compreendida como uma generalização abstrata, que apresenta uma explicação sistemática sobre as relações entre fenômenos, aplicável a uma variedade de situações reais, sendo capaz de analisar, predizer, explicar a natureza ou comportamento de um fenômeno específico. Tanto a teoria quanto o modelo permitem construir uma base mais sólida, para uma prática informada ${ }^{(6)}$.

O conhecimento dos modelos e teorias, assim como a utilização na prática, facilitará a implementação de ações eficazes de promoção da saúde, garantindo continuidade nessa

1 Enfermeira. Doutora em enfermagem. Professora do curso de graduação em enfermagem da Universidade Regional do Cariri - Urca e da Universidade de Fortaleza - Unifor. Líder do Grupo de Pesquisa Saúde Coletiva - Grupesc - Urca.

2 Enfermeira. Ph.D. Professora associada do programa de pós-graduação em enfermagem. Departamento de Enfermagem. Faculdade de Farmácia, Odontologia

e Enfermagem da Universidade Federal do Ceará - UFC. Membro do Grupo Famepe-UFC. 
direção, pois a promoção da saúde, como prática social, se faz no continuum de reflexão e ação. Quem focaliza mudanças de comportamento de saúde deve observar bases teóricas e o diálogo com as pessoas envolvidas, para criar um clima de abertura e compartilhamento no processo e resultados.

A utilização de modelos e teorias que orientem as ações de promoção da saúde para o desenvolvimento individual e coletivo contribuirá para a promoção de conhecimento, reflexão e decisão no ato de se cuidar e agir, tornar-se protagonista, além de possibilitar maiores chances no alcance dos objetivos propostos, tanto para a promoção da saúde quanto para a prevenção de doenças ${ }^{(7)}$.

Nesse entendimento, propomos a Estrutura TeóricoMetodológica de uma Participação Habilitadora, para a qual nos inspiramos no Modelo de Estágios de Mudança ${ }^{(8)}$. Por meio dessa Estrutura Teórica, examinamos a participação de usuários na Estratégia Saúde da Família (ESF), a partir da decisão individual, de uma atitude de prontidão, para agir sobre o ato de cuidar de si, na família e no grupo, como agente de mudança ${ }^{(9)}$.

Neste estudo, apresentamos a Estrutura Teórica e como ela foi utilizada para a compreensão da participação dos usuários.

\section{Apresentando a Estrutura Teórica}

A Estrutura Teórico-Metodológica de uma Participação Habilitadora foi inspirada, como referido, no Modelo de Estágios de Mudança, desenvolvido em 1982, resultado do estudo comparativo de teorias de psicoterapia e de mudança de comportamento. Ela descreve e explica diferentes estágios comuns que ocorrem com as pessoas durante os processos de mudança. Baseia-se no fato de que a mudança de comportamento é processual e que os indivíduos têm vários níveis de motivação e prontidão para mudar ${ }^{(1,10)}$.

Ao desenvolverem um instrumento para identificar os estágios de mudança, chegaram a quatro estágios definidos, confiáveis e relacionados entre si: pré-contemplação, contemplação, determinação ou preparação e ação ${ }^{(8)}$. Os autores trazem ainda o estágio denominado manutenção, que identificam como etapa de manutenção. Os estágios estão descritos na sequência:

1. pré-contemplação: nesse estágio, não existe intenção de mudança do comportamento em um futuro breve, nem mesmo a crítica a respeito do conflito envolvendo o comportamento-problema. $\mathrm{O}$ indivíduo não tem consciência do comportamento que deve ser mudado. Pode ter noção das consequências de seu comportamento, mas não planeja mudá-lo. Nessa fase, o profissional de saúde pode discutir com o indivíduo os problemas associados com a doença ou condição de risco. No estudo ${ }^{(9)}$, esse estágio foi identificado quando os usuários receberam informações para a mudança de comportamentos de saúde e, no entanto, não demonstraram uma mesma atitude para a mudança;

2. contemplação: caracteriza-se pela conscientização de que existe um problema, mas há uma ambivalência quanto à perspectiva de mudança. Nesse estágio, as pessoas estão pensando na mudança de comportamento; o profissional poderá discutir com o indivíduo os potenciais benefícios que a mudança pode trazer. Machado(9) identificou essa etapa quando houve mudança de comportamento e o reconhecimento dos benefícios ocorridos no indivíduo, mas sobretudo em relação à família;

3. determinação ou preparação: fase em que o indivíduo se compromete seriamente com as mudanças e decide fazê-las em curto período. Foi identificado esse estágio quando o usuário, além de cuidar de si e da família, passa a compreender a ação no coletivo, identificando ações necessárias no entorno ${ }^{(9)}$;

4. ação: esse estágio acontece quando o indivíduo escolhe uma estratégia para a realização dessa mudança e toma uma atitude nesse sentido. A mudança já se iniciou e o profissional elabora, com o indivíduo, um plano de ação para o indivíduo, a família e o entorno;

5. manutenção: continuidade da mudança, para benefício da saúde; nessa fase, a vontade de manter a mudança é fator-chave para o sucesso, pois é comum ocorrer recaídas. É o estágio em que se trabalham a prevenção à recaída e a consolidação dos ganhos obtidos durante a ação. $O$ profissional realiza o acompanhamento do indivíduo e as mudanças. Os dois últimos estágios de mudanças apresentados foram identificados no estudo ${ }^{(9)}$, quando o usuário, além de todas as mudanças ocorridas nos demais estágios, demonstrou interesse em participar ou participou junto à unidade de saúde/gestão.

Esse modelo é circular e tem aplicação tanto no plano individual quanto no contexto de programas. Facilita a decisão, quando as pessoas querem mudar, identifica barreiras para as mudanças, reconhecendo obstáculos em problemas comuns durante a mudança. $\mathrm{O}$ indivíduo não caminha nos estágios de forma linear-casual, ou seja, em que há uma causa, um efeito e, consequentemente, alteração no comportamento, como anteriormente se pensava. As mudanças de estágios são mais bem representadas por uma espiral, em que as pessoas podem progredir ou regredir, sem ordenação lógica(11).

Essa compreensão nos conduziu à elaboração da estrutura teórica, denominada Estrutura Teórico-Metodológica de uma Participação Habilitadora. Essa estrutura teórica foi inspirada no Modelo de Estágios de Mudança(8), no qual buscamos, a partir desse construto, identificar níveis de mudanças ocorridos nos indivíduos e na família ao longo da incorporação na ESF. Tomamos como descritores de evidência comportamentos e atitudes manifestados pelos usuários como resultado de sua participação na ESF.

A estrutura é a fundamentação conceitual de um estudo. Nem todo estudo é baseado em uma teoria ou modelo conceitual, mas todos possuem uma estrutura, que pode estar implícita ou descrita pelo pesquisador ${ }^{(6)}$. A figura 01 traz a estrutura teórico- 
metodológica, formada por níveis de participação do usuário na Estratégia Saúde da Família.

A mudança consciente ocorre com a participação efetiva do usuário/família nos meios de produção de conhecimentos e de habilidades para agir na promoção da saúde - ou seja, o que definimos como participação habilitadora. Esse tipo de participação é entendida como processo consciente, crítico, em que a mudança de comportamento para cuidar da saúde ocorre pela aprendizagem sobre saúde, pelo desenvolvimento de habilidades, pelo entendimento das condições de saúde articuladas ao estilo de vida ou ainda no modo como os serviços de saúde operam. Dessa forma, acreditamos que, a partir desse entendimento, o usuário terá de fato vivido uma participação habilitadora, no sentido de incorporar uma mudança consciente, sendo capaz de mudar, mudar na família e mudar o contexto em que vive.
A participação habilitadora, entendida como aquela que leva à autonomia e emancipação do usuário, constituída por meio do processo educativo, pode acontecer em diferentes níveis dentro da Estratégia Saúde da Família. Acreditamos que isso ocorra em movimento espiral ascendente, como demonstrado na figura 01, por meio do estímulo à participação do usuário para o desenvolvimento de conhecimentos e habilidades a fim de agir na promoção da saúde, do mais simples para o de maior complexidade. No primeiro nível, o indivíduo aprende a cuidar de si mesmo. No segundo, a cuidar dentro do contexto da família. No terceiro, a cuidar do entorno. E, por último, a exercer a cidadania.

No primeiro nível, o usuário estará apto a cuidar de si, representando, nesse caso, uma participação normativa. $\mathrm{O}$ usuário toma para si uma consciência ou necessidade para cuidar da doença que afeta a saúde com ênfase no tratamento e

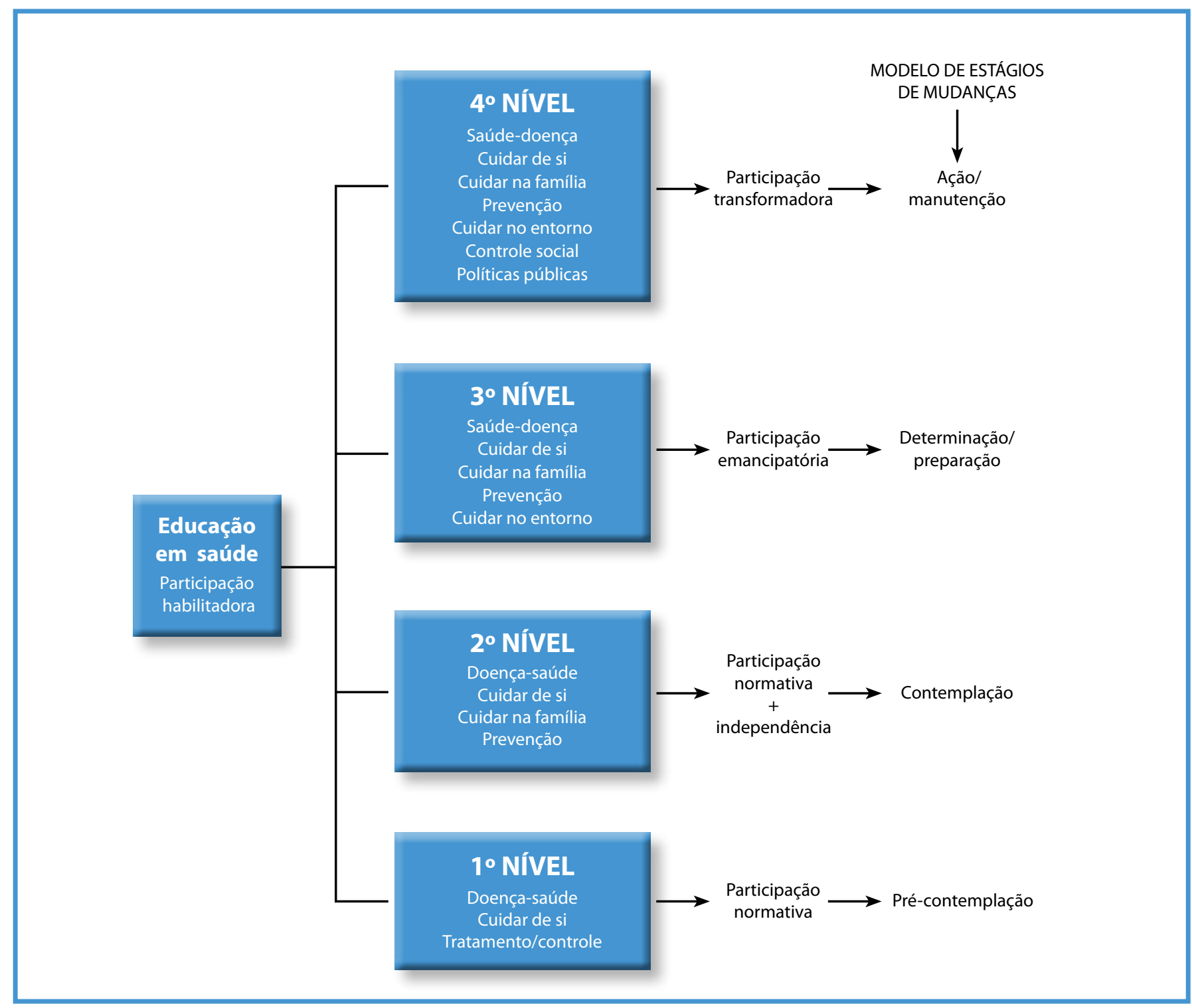

Figura 01 - Estrutura Teórico-Metodológica de uma Participação Habilitadora 
controle dessa, mas ainda está muito no plano individual. Nesse, identificamos o estágio de pré-contemplação. O indivíduo pode mudar, mas não tem nenhuma intenção de que essa mudança aconteça de imediato ou que seja mantida. Para ilustrar uma situação que se enquadre nesse nível, podemos trazer, como exemplo, a mãe que, quando o filho tem uma diarreia, aprende como conduzir essa situação em casa, utilizando o soro caseiro, mas, quando a criança contrai outra diarreia, ela logo procura o serviço de saúde. Nesse caso, não houve mudança, não se formou uma consciência para isso; o usuário tem a informação, mas não demonstra uma mudança efetiva.

No segundo nível, a participação do usuário é ampliada para o desenvolvimento de uma consciência além de cuidar de si, sobretudo para cuidar na família, considerada uma participação normativa + independência. Nesse sentido, o indivíduo toma consciência de que os problemas podem afetar a dinâmica familiar, como, por exemplo, quando é hipertenso e faz uso do álcool ou tabaco, criando conflitos e afetando o relacionamento com a família. Nesse caso, o indivíduo toma consciência de que isso o prejudica e também sua família. Nesse nível, há uma compreensão do usuário de que, quando algo o afeta ou a um membro da família, toda a família, de alguma forma ou em alguma dimensão, é afetada. Nesse caso, podemos considerar que houve um estágio de contemplação.

No terceiro nível, o usuário, além de cuidar de si e da família, estendea participação para cuidar de seu entorno, oque representa o início da formação de uma consciência crítica de cuidado coletivo, representando uma participação emancipatória. Os problemas do grupo e/ou da comunidade passam, sobretudo, a tornar-se seus problemas, daí o envolvimento no sentido de solucioná-los. Para ilustrar esse nível, podemos pensar numa situação que afeta diretamente o indivíduo e também a comunidade, como, por exemplo, a falta de profissionais ou medicamentos no serviço para um cliente hipertenso. $O$ cliente passa a ter consciência de que não é só ele o afetado, mas todo um grupo que é hipertenso como ele. Há uma tomada de consciência de que, além de cuidar de si e da família, ele faz parte de um grupo. Ocorre assim o estágio de determinação ou preparação.
No quarto nível, o usuário cuida de si, da família e de seu entorno e há compreensão e envolvimento com as políticas públicas, aqui representadas pela participação do usuário nos conselhos municipais de saúde, mobilizações sociais e outros grupos, constituindo uma participação transformadora. Nesse nível, o indivíduo passa a perceber que o problema não é só do grupo de hipertensos ou dos diabéticos, por exemplo, mas de toda uma coletividade, decorrendo daí a necessidade do envolvimento da sociedade como um conjunto para solucionálo. O usuário será capaz de buscar em um contexto mais amplo com demais grupos, juntando-se com outros movimentos sociais e estratégias para viabilizar ações de promoção da saúde na realidade na qual se encontra. Consideramos que acontece o estágio de ação e/ou de manutenção.

\section{A visão acerca da estrutura teórica}

O uso de referencial teórico auxilia na compreensão dos objetos de investigação e amplia o olhar do pesquisador na análise do fenômeno.

A estrutura construída e utilizada no estudo ${ }^{(9)}$ auxiliou na compreensão da participação do usuário dentro da Estratégia Saúde da Família, no sentido de apontar, por intermédio dos estágios de mudança, qual a direção político-ideológica que o movimento educativo dentro da ESF conduz quanto à participação do usuário.

O uso dessa Estrutura Teórica poderá ser um caminho a percorrer para a construção de um conhecimento que possibilite compreender como a Estratégia Saúde da Família transforma a vida das pessoas, e, mais ainda, o modo de participar e cuidar da saúde. Entendemos que a ESF, como estratégia estruturante de fortalecimento do SUS, terá sua filosofia concretizada quando efetivar a participação plena do usuário; ou seja, ensejando nas pessoas o desenvolvimento de uma participação habilitadora, que resulte num processo emancipatório, tornando visível e permeável o controle social.

Pensamos que essa Estrutura Teórica pode ser usada em outros estudos, em cenários distintos dos aqui apontados, investigando diferentes atores em experiências de participação.

\section{Referências}

1. Naidoo J, Wills J. Health promotion - foundations for practice. London:

Baillière Tindal/Royal College of Nursing; 1994.

2. Riehl JP, Roy C, editores. Conceptual Model for Nursing practice. Norwalk: Appleton-Century-Crofts; 1980.

3. Chinn PL, Kramer MK. Theory and nursing: a systematic approach. $4^{\mathrm{a}}$ ed. St. Louis: Mosby; 1995.

4. Barnum BS. Nursing theory: analysis, application, evaluation. $5^{\mathrm{a}}$ ed. [s.I.]: Lippincott; 1998.

5. George JB. Nursing theorises: the base for profissional nursing practice. New Jersey: Upper Saddle River; 2002.

6. Polit DF, Beck CT, Hungler BP. Fundamentos de pesquisa em enfermagem: métodos, avaliação e utilização. 5ª ed. Porto Alegre: Artmed; 2004.
7. Freudenberg N, Eng E, Flay B, Parcel G, Rogers T, Wallersterin NS. Individual and community capacity to prevent disease and promote health: searching of relevant theories and principles. Health Edu Q. 1996;22(3):290-306. 8. Prochaska JO, Diclemente C. Transtheorical therapy: toward a more integrative model of change. Psycoth theory res pract. 1982;20:161-73. 9. Machado MFAS. Compreensão das mudanças comportamentais do usuário no PSF por meio da participação habilitadora [tese]. Fortaleza: Faculdade de Farmácia, Odontologia e Enfermagem da Universidade Federal do Ceará; 2007.

10. Bunton R, Macdonald G. Health promotion. New York: Routledge; 1992. 11. Prochaska JO, Diclemente C, NorcrosS JC. Search of how people change: applications to addictive behaviors. Am Psychol.1999;47:1102-13. 